menstruum of the tincture. As one of the objects of this research was to conserve alcohol it was particularly gratifying to note that a tincture made with a menstruum of $65^{\circ}$ parts of alcohol and $35^{\circ}$ parts of water produced a preparation that was all that could be desired both from a pharmaceutical and a therapeutic standpoint. The alkaloidal content was as required by the Pharmacopoeia. That this menstruum was sufficient to extract all the alkaloids of this drug was shown by the failure of the mare to give any reaction with Mayer's reagent after further extraction.

Depriving the drug of its oil in no way depreciates its activity, as the oil therefrom gave no evidence of containing any alkaloid.

\title{
THE ANALYSIS OF MERCURIAL, OINTMENT.*
}

BY JOSEPH L. MAYER.

The official method for the assay of Mercurial Ointment being too inaccurate and time consuming, $I$ devised the following simpler and more accurate procedure.

Accurately weigh about 0.500 gramme of the ointment into a tared roo-Cc. beaker, warm gently; add about $50 \mathrm{Cc}$. of gasoline, stir with a glass rod to dissolve the fat, allow the mercury to subside, then carefully decant the liquid through a small filter paper and reject filtrate. Now place funnel and filter paper over the beaker containing the mercury; add ro $\mathrm{Cc}$. nitric acid through the paper, employing a pipette; wash with a small amount of water, and then place the beaker containing the mercury and nitric acid on a hot plate and heat gently until red fumes cease to be evolved; wash the contents into a large Erlenmeyer flask, dilute to about $x_{5} \mathrm{Cc}$. with water, add $2 \mathrm{Cc}$. ferric ammonium sulphate test solution and titrate with tenth-normal KCNS V.S. until the appearance of a permanent, yellowishred color.

Each Cc. of tenth-normal KCNS V.S. consumed corresponds to 0.01003 gramme of mercury.

Three assays made of a sample of blue ointment by this process yielded the following results: $30.034,30.234$ and 30.157 percent of mercury.

The accuracy, simplicity, and rapidity of this method commend it for inclusion in the Pharmacopoeia.

RESEARCH AND ANALYTICAI. LABORATORIES, OF THE

LouIs $\mathrm{K}$. LIGGET'T Co.

\section{TINCTURE OF VANILLA. ${ }^{1}$}

BY K. A. BARTLETT.

While tincture of vanilla is a preparation that has been in use for a great many years, there has never been a satisfactory official formula for it. At the time of the last revision of the U. S. P. the formula therein was not satisfactory. A new formula was devised and included in the N. F. IV.

* Read before Scientific Section A. Ph. A., city of Washington meeting, rgzo.

I Presented before Section on Practical Pharmacy and Dispensing, A. Ph. A., City of Washington meeting, r920. 
This formula, however, instead of being an improvement, was decidedly a step in the wrong direction. It is troublesome enough on a small scale and as a manufacturing proposition it is almost impossible. One of the faults common to the formulae of both the U. S. P. VIII and the N..F. IV is the fact that they direct mixing the sugar with the cut vanilla beans and then direct percolation of this mixture. The N. F. IV in addition to this fault, unnecessarily complicates matters by first macerating the cut beans with straight alcohol. This alcohol is then filtered off and diluted with an equal volume of water, this mixture then being used as a menstruum for the final percolation. In order to bring out the fault with this manner of manipulation it may be well to refer back to the actual preparation of these beans for market, where just prior to being tied into bundles and wrapped, they are given a thin coating of a fixed oil. This, together with the small amount of fixed oil that is naturally in the beans, readily explains why the above procedure is unsatisfactory. The initial maceration with straight alcohol dissolves the fixed oil and then on diluting the percolate with water an unsightly mixture results, due to the throwing of this fixed oil out of solution. When this mixture is poured on the percolator, containing the very viscid mixture of sugar and cut vanilla beans, the percolator rapidly clogs and renders percolation so difficult that it is impractical.

The following formula is submitted as one which has been in use for some time and one which gives very satisfactory results:

$\begin{array}{lr}\text { Vanilla, cut in small pieces } & \text { I00 Gm. } \\ \text { Sugat, granular } & 200 \mathrm{Gm} . \\ \text { Dilute alcohol, a sufficient quantity to make } & \text { I000 mils. }\end{array}$

Place the cut vanilla beans together with three hundred and twenty mils (320 mils) of dilute alcohol into a rooo-mil flask, preferably one with a wide mouth, and heat on a water bath under a reflux condenser for 6 hours, keeping the mixture nearly to the boiling point, but not high enough to cause any loss of alcohol. Allow to cool and strain the liquid through cheesecloth.

Then place the cut vanilla beans in a percolator, preparing the percolator as follows:-

In the bottom place a piece of filter paper large enough to cover the bottom and extend a short way up the sides of the percolator.

Then put in a layer of clean white sand, on top of that a layer of kieselguhr and then another layer of sand. Pack alternately a layer of vanilla beans and a layer of kieselguhr.

Pour on the strained liquid and percolate slowly, continuing percolation with diluted alcohol. Mix the sugar with the percolate as fast as it is received and dissolve by agitation. Continue percolation to obtain 1000 mils.

The continuous heating of the beans with a portion of the menstruum greatly facilitates extraction. There is no unsightly and troublesome precipitation of fixed oil and percolation is easy.

Anyone who has had experience with tincture of vanilla knows the difficulties attending its filtration and to overcome this I strongly recommend that a percolator prepared in the above described manner be used. Any excess of oil which may be extracted from the drug is immediately removed by the kieselguhr and sand, and the percolate comes brilliantly clear. If a good quality, clean sugar be used, filtration will be unnecessary.

In manufacturing the preparation on a larger scale the cut vanilla beans and 
diluted alcohol may be placed in a clean still of suitable capacity, the lid tightly clamped down and then the mixture heated for the specified length of time.

The advantages of this formula over the present one are many, and I strongly recommend its adoption to.replace the formula now official.

LABORATORIES OF

E. R. SQuire \& SoNs,

BROOKLYN, N. Y.

\section{ISO-ALCOHOLIC ELIXIRS.}

BY BERNARD FANTUS.*

Aromatic elixir, which is intended to serve as a vehicle for alcoholic preparations and alcohol-soluble medicaments, falls short of its purpose by reason of its fixed alcohol content, which makes it suitable as a diluent only for hydro-alcoholic tinctures of rather low alcohol percentage, and as a vehicle for drugs that do not require strong alcohol for solution. This difficulty may, of course, easily be overcome by fortifying the elixir with alcohol, so as to give it the alcoholic strength required. It is, however, practically impossible for the prescribing physician to carry in his mind the alcoholic strength of the various menstrua used in the extrac tion of the drugs he employs, and it is not always possible for him to consult a reference book. The pharmacist, on the other hand, could easily adjust, with the aid of his official books, the proportion of alcohol in the elixir, so as to make it compatible with the medicament it is to carry. Indeed, this ought to be required of the pharmacist by making the aromatic elixir of the Pharmacopoeia "iso-alcoholic," $i . e$. , of an alcoholic strength adjustable to the requirements of the medicament for which it is to serve as a vehicle.

This would not make it necessary for the druggist to carry in stock elixirs of varying alcoholic strengths; as he could easily, by means of a table, adjust the strength of a standard elixir to the alcohol percentage demanded. With this in view, it might be well to reduce the alcoholic strength of aromatic Flixir to five percent, which is that of some of the "aqueous" elixirs of the National Formulary, such as the Compound Elixir of Almond, and the Aqueous Elixit of Glycyrrhiza. This reduction would have several advantages. The "aqueous" elixir would be much less likely to be sought after as an alcoholic beverage. Furthermore, it would reduce the expense of quite a number of preparations, such as the bromide elixirs and many other elixir solutions of water-soluble chemicals of the N. F., the alcohol contents of which are at present decidedly excessive.

The following modification of the official formula for aromatic elixir might therefore be proposed:

AROMATIC ELIXIR.

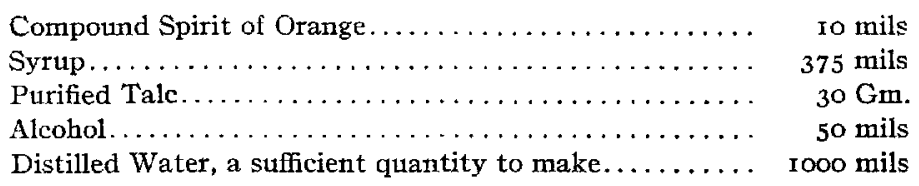

Mix the compound spirit of orange with the alcohol. To this solution add the syrup in several portions, agitating after each addition, and afterwards add in the same manner 560 mils

* Associate Professor of Therapeutics, Rush Medical College. 